\title{
Searching for Lightweight Dark Matter in NOvA Near Detector
}

\author{
Filip Jediný* \\ Czech Technical University in Prague \\ Brehova 7, Prague, Czech Republic \\ E-mail: filip.jedinyefjfi.cvut.cz
}

\section{Athanasios Hatzikoutelis}

University of Tennessee Knoxville

Knoxville, TN, USA

E-mail: a.hatzikoutelis@utk.edu

\section{Sergey Kotelnikov}

Fermi National Accelerator Laboratory

Kirk and Pine st., Batavia, IL, USA

E-mail: kotelnik@fnal.gov

\section{Biao Wang}

Southern Methodist University

Dallas, TX, USA

E-mail: biaow@mail.smu . edu

\begin{abstract}
The NOvA long-baseline neutrino oscillation experiment is receiving record numbers of $120 \mathrm{GeV}$ protons on target from Fermilab's NuMI neutrino beam. We take advantage of our experiment's sophisticated particle identification algorithms to search for Lightweight Dark Matter (LDM) in the first year of data from the Near Detector of NOvA (300-ton low-Z mass, placed off the beam axis) during the experiment's first physics runs. Theoretical models of LDM predict that bellow$10 \mathrm{GeV}$ candidates produced in the NuMI target might scatter or decay in the NOvA Near Detector. We simulate an example of the Neutral Vector Portal model with the sensitivity estimate of $10^{-39}$ $\mathrm{cm}^{2}$, which corresponds to $O(10)$ LDM candidates per three years of data, looking at single electromagnetic showers between 5 and $15 \mathrm{GeV}$ in a model independent way.
\end{abstract}

The 15th International Conference on Flavor Physics \& CP Violation

5-9 June, 2017

Prague, Czech Republic

\footnotetext{
*Speaker
} 


\section{Introduction}

There have been several ideas proposing a search for Dark Matter candidates from so called Hidden sector [1,2]. We take the advantage of the accelerator technology developed for Intensity Frontier, which, according to some models [2-6], might be capable of producing mediators feebly coupling those HS candidates to SM (so we can detect them). A Neutral Vector Portal model predicts direct (quark on quark) or indirect (meson decay) production of $V$ mediators at the high luminosity proton beam-dumps, decaying in a pair of Dark Matter particles. The cosmological constrains estimate $\sim 40 \mu \mathrm{b}$ production of $1 \mathrm{GeV}$ vector particle $V$ at NuMI beam [7], decaying to DM particles which might then interact in a near detector through a reverse process, scattering on a nucleon or electron of a neutrino detector.

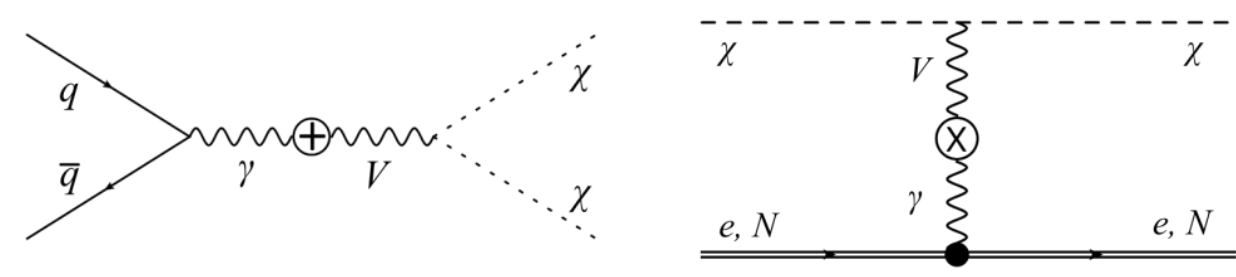

Figure 1: Direct production of vector mediator $V$ and DM interaction via the reversed process.

\section{NOvA ND as a beam-dump experiment}

The NOvA (NuMI Off-axis electron-neutrino Appearance) experiment at Fermilab uses the world's most intensive beam, the NuMI, with bunches of $120 \mathrm{GeV}$ primary protons striking a carbon target at an intensity of up to $700 \mathrm{~kW}$. The NOvA Near Detector sits underground, one kilometer from the target area, shielded by the surrounding rock, leaving only neutrinos (and potentially other rarely interacting particles) to reach the detector. Expectedly, any LDM induced events would be very rare compared to the 6-order of magnitude more $\mathrm{CC}$ neutrino interactions on nucleons, or the 4-orders of magnitude higher-rate NC.

The NOvA Near Detector is positioned 11 meters to the left of the beam center (14 mrad off-axis, less than $1^{\circ}$ ), which defines the beam-dump re-interpretation of the complex. The beam neutrinos at this angle have a narrow band of energies peaking around $2 \mathrm{GeV}$ [7] and travel mostly through the earth that protects the NOvA ND from all high energy hadronic products much better than other current setups. The expected neutrino-induced background rate per year is of the order of $10^{6}$ up to $3 \mathrm{GeV}$ and drops by two orders at $5-10 \mathrm{GeV}$ and by 2 more orders in the region above $30 \mathrm{GeV}$. The main expectation for a viable measurement is that the real LDM rate drops off slower with energy and therefore, after the total data accumulation and the event type identification, the LDM signal to neutrino background will improve.

\section{LDM from NuMI model example}

Reinterpretation of the NuMI source and the NOvA ND complex as a beam-dump experiment enables searches of rare events in a model independent way. For the popular example of the Vector Portal, the mass of a directly produced vector mediator particle may reach maximum of $15 \mathrm{GeV}$ available from the $120 \mathrm{GeV}$ of the proton on the carbon target. The studies with the NOvA ND on LDM masses can extend up to $7.5 \mathrm{GeV}$. This range of LDM masses cannot be accessed by direct detection experiments as they have a cut-off above the $10 \mathrm{GeV}[8]$ and it cannot be studied 
either by other current fixed target neutrino experiments as they have lower proton beam energy and therefore, cannot produce mediators heavier than $1 \mathrm{GeV}[8,9]$.

The limits on sensitivity are calculated using the PYTHIA 8.1 framework [10] on a simplified case of direct production of a $1 \mathrm{GeV}$ vector mediator decaying instantly to a pair of LDM with masses of 100, 300 and $450 \mathrm{MeV}$.

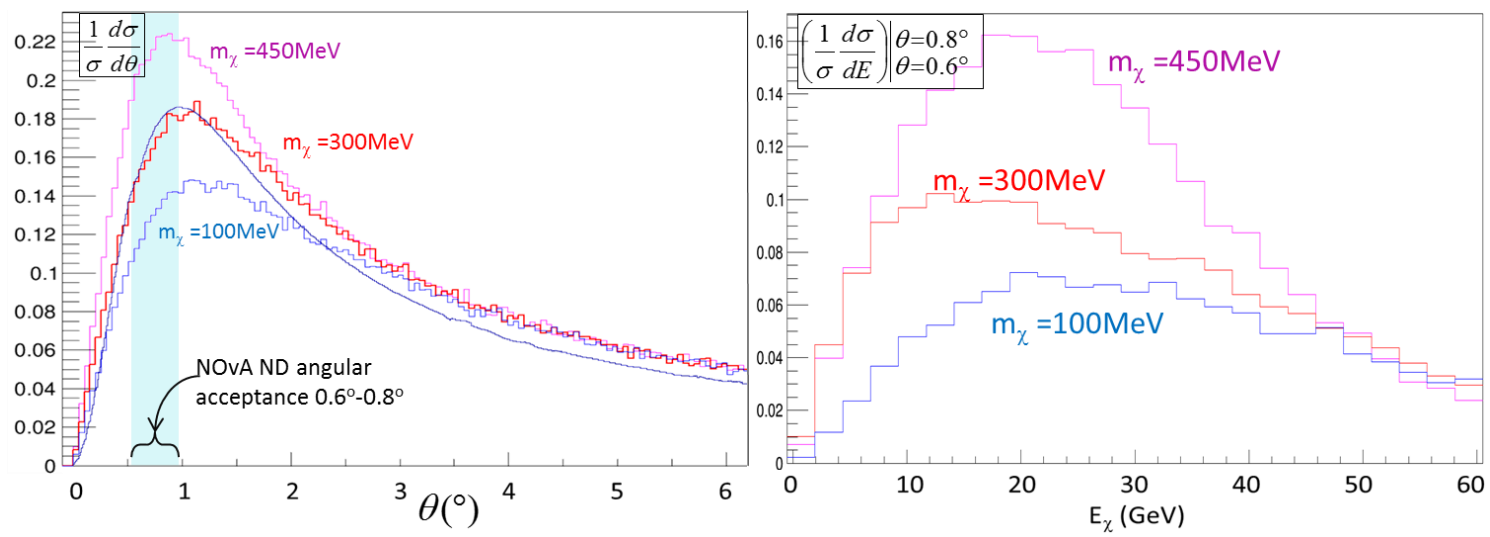

Figure 2: The simulated normalized LDM angular distributions and the LDM kinetic energy spectra within the ND acceptance range of $0.2^{\circ}$.

\section{LDM interactions in NOvA ND}

At NuMI beam intensity of $\sim 6 \cdot 10^{20}$ POT per year, the expected production rate is estimated to $O\left(10^{14}\right)$ LDM particle candidates per year, leaving the target area boosted forward into few degrees around the beam direction.

NOvA Simulation

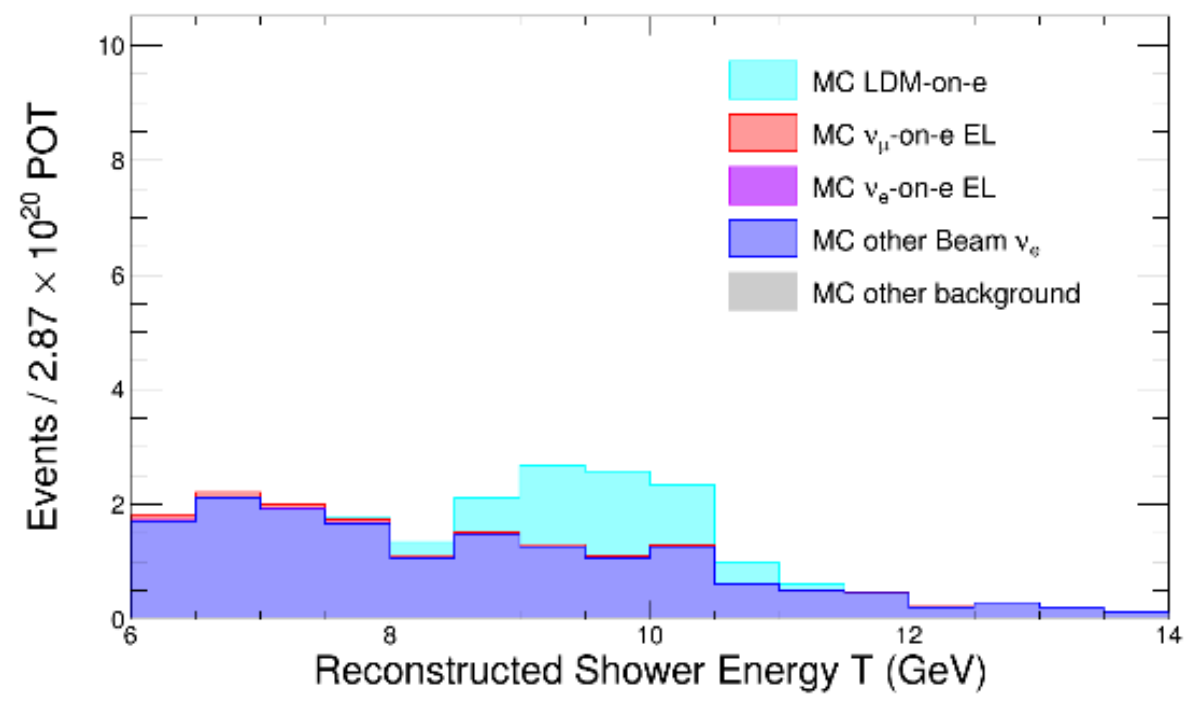

Figure 3: The single EM-showers in the NovA ND background analysis.

The angular acceptance of NOvA ND is $0.6^{\circ}<\theta<0.8^{\circ}$ and the energy distribution from few GeV up to $60 \mathrm{GeV}$ peaks around $20 \mathrm{GeV}$, much higher than the neutrino $2 \mathrm{GeV}$ maximum.

The low intensity interaction example for this work is the cleanest of discussed channels - the elastic scattering of LDM on atomic electrons, a neutral current event dependent on the kinematics of the involved particles only. This channel is relatively weak compared to the scattering on nucleons but it is a clear, well-understood, leptonic process, with an easily identifiable single 
electromagnetic shower along the LDM direction pointing directly back to the target, without any hadronic effects. However, the EM showers of higher energies may not be contained in the 330ton NOvA ND, so the identification efficiency declines with higher energies.

The scattering cross section is between $10^{-36}$ and $10^{-45} \mathrm{~cm}^{2}$ (from direct detection experiments and several collider monojet measurements), resulting in up to thousands of events per year.

For comparison, the EM spectra induced by neutrino interactions were produced by the NOvA analysis framework, using a sophisticated artificial neural network PID SW, which was tuned for energies within 1 and $3 \mathrm{GeV}$. The search area for the LDM signal extends from 5 to $15 \mathrm{GeV}$, since the PID tools are inefficient beyond $20 \mathrm{GeV}$. We have used the same PID methods and cuts on the first-year neutrino data and custom LDM signal files. All the expected neutrino interaction channels that have similar signatures are the background for the LDM signal (Figure 3). At this low exposure, statistical errors and uncertainties in target-kaon production would dominate the measurement.

This novel discovery approach with existing NOvA detector could become a precision measurement with future projects like DUNE [11] and SHiP [12], using higher intensities, energies and fiducial volumes at shorter baselines.

\section{References}

[1] Rouven, E. et al. Dark Sectors and New, Light, Weakly-Coupled Particles. hep-ph. 2016, arXiv:1311.0029.

[2] Batell, B. et al. (Project X). New, light, weakly-coupled particles with Project X. FERMILAB-TM. 2013, chapter VII, 2557.

[3] de Niverville, P., Pospelov, M., Ritz, A. Observing a light dark matter beam with neutrino experiments. Phys. Rev. D. 2011, Vol. 84, arXiv:1107.4580.

[4] Lin, T., Yu, H., Zurek, K. M. Symmetric and asymmetric light dark matter. Phys. Rev. D. 2012, Vol. 85, arXiv:1111.0293.

[5] de Niverville, P., McKeen, D., Ritz, A. Signatures of sub-GeV dark matter beams at neutrino experiments. Phys. Rev. D. 2012, Vol. 86, arXiv:1205.3499.

[6] de Niverville, P. Searching for Sub-Gev Dark Matter at Fixed Target Neutrino Experiments. Physics Procedia. 2014, Vol. 61.

[7] Abramov, A. G. et al. Beam optics and target conceptual designs for the NuMI project. Nucl.Instrum.Meth. 2002, Vol. A485.

[8] Aprile, E. et al. (XENON100 Collaboration). Dark Matter Results from 225 Live Days of XENON100 Data. Phys. Rev. Lett. 2012, Vol. 109, arXiv:1207.5988.

[9] Kelso, Ch., Hooper, D. and Buckley, M. R. Toward a consistent picture for CRESST, CoGeNT, and DAMA. Phys. Rev. D. 2012, Vol. 85, arXiv:1110.5338.

[10] Sjöstrand, T., Mrenna, S., Skands, P. A Brief Introduction to PYTHIA 8.1. Comput.Phys.Commun. 2008, Vol. 178, arXiv:0710.3820.

[11] Acciarri, R. et al. Long-Baseline neutrino Facility. FERMILAB-DESIGN-2016-02. 2015, Volume 2: The Physics Program for DUNE at LBNF, arXiv:1504.04956.

[12] Anelli, M. et al. (SHiP Collaboration). A facility to Search for Hidden Particles (SHiP) at the CERN SPS. CERN-SPSC-2015-016. SPSC-P-350, 2015, arXiv:1504.04956. 